\title{
The Regulation and Expression of the Creatine Transporter: A Brief Review of Creatine Supplementation in Humans and Animals
}

\author{
Ryan D. Schoch, Darryn Willoughby, and Mike Greenwood. \\ Exercise and Biochemical Nutrition Laboratory, Baylor University, Waco, TX. Address \\ correspondence to Ryan_Schoch@baylor.edu.
}

Received February 28, 2006/Accepted June 21, 2006

\begin{abstract}
Creatine monohydrate has become one of the most popular ergogenic sport supplements used today. It is a nonessential dietary compound that is both endogenously synthesized and naturally ingested through diet. Creatine ingested through supplementation has been observed to be absorbed into the muscle exclusively by means of a creatine transporter, CreaT1. The major rationale of creatine supplementation is to maximize the increase within the intracellular pool of total creatine (creatine + phosphocreatine). There is much evidence indicating that creatine supplementation can improve athletic performance and cellular bioenergetics, although variability does exist. It is hypothesized that this variability is due to the process that controls both the influx and efflux of creatine across the cell membrane, and is likely due to a decrease in activity of the creatine transporter from various compounding factors. Furthermore, additional data suggests that an individual's initial biological profile may partially determine the efficacy of a creatine supplementation protocol. This brief review will examine both animal and human research in relation to the regulation and expression of the creatine transporter (CreaT). The current literature is very preliminary in regards to examining how creatine supplementation affects CreaT expression while concomitantly following a resistance training regimen. In conclusion, it is prudent that future research begin to examine CreaT expression due to creatine supplementation in humans in much the same way as in animal models. Journal of the
\end{abstract} International Society of Sports Nutrition. 3(1): 60-65, 2006.

Key Words: creatine monohydrate, sports supplements, ergogenic aids

\section{INTRODUCTION}

Creatine monohydrate, or methyl guanidineacetic acid, has become one of the most popular ergogenic sport supplements used today. Creatine was first discovered in 1835 by a French scientist, Chevreul, followed by the first research trials occurring within the early 1900's on the fate of administered creatine ${ }^{1}$. At this time both humans ${ }^{2}$ and animals ${ }^{3}$ were studied but it wasn't until the 1990's that it was finally determined that creatine supplementation increased the pool of metabolically-active creatine in muscle ${ }^{4}$. Once the physiological relevance of the creatine-phosphate bioenergetic pathway was discovered, much research began exploring how creatine supplementation may enhance athletic performance. Although beyond the scope of this review, it should also be noted that creatine supplementation may also benefit individuals diagnosed with various neuromuscular disorders and medical conditions.

\section{CREATINE METABOLISM}


Creatine is a nonessential dietary compound that is both endogenously synthesized, primarily in the liver, and naturally ingested through omnivorous diets, with the greatest natural quantity of creatine present in red meats. Creatine synthesized from the liver is released into the bloodstream and then taken up by muscle fibers predominately by way of a sodium-chloride dependent creatine transporter, CreaT1 ${ }^{5}$. There are actually two isoforms of creatine transporters, CreaT1 and CreaT2, of which the latter is primarily active and present within the testes ${ }^{6}$. Creatine ingested through supplementation has been observed to be absorbed into the muscle exclusively by means of CreaT1. Therefore, creatine transporter discussion during the remainder of this manuscript will refer to CreaT1 as CreaT, since muscle fibers are of the greater focus.

It has been observed that creatine uptake is regulated by a variety of mechanisms. Speer et al. discussed that phosphorylation and glycosylation of the creatine transporter, in addition to changes in the extracellular and intracellular creatine content, may result in a means of regulation of the CreaT protein, which in turn, would affect creatine uptake rates ${ }^{7}$. Walzel et al. observed that there may be an existence of not only cytosolic, but also a mitochondrial creatine pool, through the observance of CreaT isoforms within the mitochondria ${ }^{8}$. These researchers concluded that the mitochondria "may represent a major compartment of creatine transporter localization, thus providing a new aspect to the current debate about the existence and whereabouts of intracellular creatine and $\mathrm{PCr}$ compartments."

\section{CREATINE SUPPLEMENTATION}

The major rationale of creatine supplementation is to maximize the increase within the intracellular pool of total creatine (creatine + phosphocreatine). The intracellular concentration of phosphocreatine ( $\mathrm{PCr}$ ) plays a significant role during the immediate bioenergetic system, which is most active during exercise at high intensity, short duration, and repeated bouts of physical activity. Through the depletion of intracellular $\mathrm{PCr}$ stores, the intracellular concentration of adenosine triphosphate (ATP), a vital molecule necessary for muscle contraction, is maintained and replenished. This occurs via a freely reversible reaction in which $\mathrm{PCr}$ phosphorylates adenosine diphosphate (ADP) to replenish ATP stores, catalyzed via the enzyme, creatine kinase. $\mathrm{PCr}$ levels within the muscle are almost 3 to 4 times more abundant than intramuscular ATP stores. While PCr is more copious than ATP, the rate in which ATP is utilized is likely to exceed the overall energy substrate regeneration necessary at activities of high intensity. However, the $\mathrm{PCr}$ supply is sufficient in providing a temporary ATP source until other bioenergetic systems reach maximal rates.

There is much evidence indicating that creatine supplementation can improve athletic performance and cellular bioenergetics. Within the literature, the most common creatine supplementation dosing regimen, indicating a significant increase in intracellular $\mathrm{PCr}$, is a loading phase of approximately $20 \mathrm{~g}$ /day for 5-7 days which is usually followed by a maintenance phase of $5 \mathrm{~g}$ /day for a period of several weeks ${ }^{26}$. However, this absolute dosing regimen may not be best. Instead, a relative amount should be used, based on either total body mass or fat free mass that yields approximately $20 \mathrm{~g} /$ day (e.g. $0.3 \mathrm{~g} / \mathrm{kg} /$ day for a $70 \mathrm{~kg}$ individual). This relative dosing regimen is based on the premise that creatine uptake will most likely differ in regards to differences in muscle mass.

Nevertheless, independent of which dosing strategy is followed, some researchers have observed no improvement in either increasing intramuscular creatine or performance measures via creatine supplementation. It is hypothesized that this variability is due to the process that controls both the influx and efflux of creatine across the cell membrane, and is likely due to a decrease in activity of CreaT from various compounding factors, which will be discussed later in this review.

\section{ANIMAL RESEARCH}

In order to begin discovering exactly how CreaT is regulated, animal research, primarily in rats, was studied through creatine supplementation. 
Guerrero-Ontiveros and Wallimann ${ }^{9}$ examined rats that were treated with a creatine analogue, $\beta$-Guanidinoproprionic acid ( $\beta$-GPA), which acts to deplete the intracellular creatine pool. $\beta$ GPA competitively competes with both creatine uptake and creatine kinase activity. Following $\beta$ GPA treatment, creatine transport activity actually increased, resulting in an increased uptake of supplemented creatine ${ }^{9}$. Additionally, it appears that creatine uptake is optimized by use of a "transport site specifically adapted to interact with an amidine group ${ }^{10}$." GuerreroOntiveros and Wallimann also discovered that when the rats were supplemented with creatine for 3-6 months, a reduced expression of creatine transporter isoforms and a reduced creatine uptake occurred ${ }^{9}$. These findings suggest that when extrapolated to human athletes that it would be: 1) undesirable to consume creatine for an extended amount of time to avoid downregulation of the creatine transporter; and 2) it would also be advisable to avoid consuming extremely high doses of creatine, as this would likely down-regulate creatine transport over time. However, the dosage used within the rats has been reported to be much greater than when applied to humans. Therefore, it is our view that the results of this study should be interpreted with caution, as the lower typical dosing regimens in humans may not display such a marked decline in creatine uptake or CreaT down-regulation through the typical moderate doses of creatine supplementation.

Murphy et al. examined how CreaT mRNA, CreaT protein, and total creatine (TCr) content varies between oxidative and glycolytic muscle fibers ${ }^{11}$. Muscle sampling, enzymatic assays, immunoblotting, immunohistochemistry, and real-time PCR were utilized to gather the data. The results indicated that $\mathrm{TCr}$ content was significantly greater in glycolytic (white) muscle fibers than in the oxidative (red) muscle. In contrast, CreaT protein content was greatest in oxidative muscle when compared to glycolytic fibers. With all muscle types, CreaT protein content was situated predominately at the sarcolemma, with evidence that some of the protein was also located internally. Lastly, realtime PCR indicated that no difference was observed in regards to the expression of CreaT
mRNA between all fiber types. This data suggests that oxidative muscle has an augmented ability to transport creatine, due to a greater CreaT protein content and a decreased $\mathrm{TCr}$ intracellular quantity. The authors also discussed that it is most likely that intracellular creatine rather than $\mathrm{PCr}$ concentration that determines the regulation of creatine uptake and CreaT activity and expression. It is plausible that as free intracellular creatine content increases, AMP-activated protein kinase (AMPK) will "initiate a signaling pathway leading to alterations in gene expression."

Wang et al. discussed that the CreaT functions similarly to neurotransmitter and amino acid transport classifications ${ }^{12}$. Since tyrosine phosphorylation is a primary mechanism in which neurotransmitter transport occurs, and that phosphorylation is known to regulate sodium-potassium-ATPase activity, creatine transport may be resultantly affected. Therefore, these researchers examined whether changes in the intracellular levels of free $\mathrm{Cr}$ via supplementation are coupled with tyrosine phosphorylation of the CreaT. The authors investigated this mechanism during sepsis; although this is beyond the scope of this review, it is interesting that oral creatine supplementation decreased tyrosine phosphorylation of the CreaT.

The literature discussed thus far dealt primarily with how the CreaT is regulated after creatine has been absorbed into the bloodstream. Peral et al. took a different approach by examining intestinal CreaT activity following supplementation ${ }^{13}$. Results from this research indicate that not only is the CreaT sodium dependent, but it is also partially chloride dependent. It was observed that increasing the chloride concentration significantly increased creatine uptake. The authors note that prior to this study, only sodium dependence was focused upon, with no evaluation of chloride's role. It is estimated that two sodium molecules and one chloride molecule are necessary for creatine transport. An additional finding concluded that $\beta$-GPA inhibited intestinal absorption most when compared to other creatine uptake inhibitors. 
An individual's muscle fiber type may also dictate the response of creatine uptake ${ }^{14}$. Brault and Terjung ${ }^{14}$ examined rat hindlimbs and their response to creatine supplementation. Creatine uptake rates and CreaT protein content were observed to be greatest in highly oxidative muscle and lowest in glycolytic fibers, whereas, CreaT mRNA was not significantly different between all fiber types. It was also found that creatine uptake was depressed in rats having a lower creatine and sodium concentration. Lastly, creatine uptake was inversely related to intracellular creatine content.

Another study conducted by Brault et al. ${ }^{15}$ evaluated how CreaT expression and the rate of creatine uptake were affected by a "long-term altered creatine environment designed to supplement, deplete, and replete intracellular total creatine ${ }^{15}$." It was hypothesized that creatine supplementation would result in both a decreased uptake of creatine and CreaT expression, whereas the utilization of $\beta$-GPA would result in vice versa. The results indicated that changes within the intracellular creatine levels significantly affect muscle fibers portraying more oxidative properties when intracellular creatine levels are low. For example, CreaT protein expression was increased following creatine depletion with the most significant changes in oxidative muscle fibers. In contrast, during repletion, lower creatine uptake rates occurred with no change in CreaT protein content. The authors speculated that the regulation of creatine uptake is possibly affected by other mechanisms than the expression of CreaT. Since the research protocol implemented high creatine feeding prior to depletion, this may have resulted in a reduction in CreaT protein preceding repletion. The results from this study have provided a potential hypothesis when applied to humans: 1) During creatine supplementation, creatine uptake would most likely be more pronounced in individuals possessing the lowest initial intracellular creatine concentration; and 2) Humans with a lower intracellular creatine level would not be expected to uptake creatine as effectively. Both of these scenarios assume that all individuals obtained similar plasma creatine levels from creatine supplementation. This warrants future research since, unfortunately, most creatine supplementation research uses an absolute dose rather than a relative quantity based upon total body mass or fat-free mass.

\section{HUMAN RESEARCH}

Human research on CreatT1 expression has been fairly limited to health and disease issues ${ }^{16-19}$. However, some studies have examined how $\mathrm{Cr}$ supplementation in humans affects CreaT gene expression in vegetarians ${ }^{20}$, males versus females ${ }^{21}$, and between young and elderly individuals ${ }^{22}$.

In regard to individuals diagnosed with neuromuscular disorders, it has been observed that total creatine and $\mathrm{PCr}$ levels are decreased in myopathies ${ }^{23}$. Tarnopolsky et al. selected patients portraying this diagnosis and measured skeletal muscle CreaT protein in addition to sarcomeric mitochondrial creatine kinase protein content $(\mathrm{mtCK})^{18}$. CreaT protein content was significantly lower for those with myopathies. However, mtCK varied between participants. These results indicate that lower levels of CreaT protein is the major contributor to decreased total creatine and PCr levels in myopathies, and that creatine supplementation may provide a beneficial treatment to restore these stores.

Pyne-Geithman et al. conducted a descriptive case study on an individual that carried a mutation of the X-linked creatine-transporter gene ${ }^{16}$. This type of mutation generally results in virtually complete creatine depletion in the brain, although observed musculoskeletal and cardiac function remain as normal. A muscle biopsy was excised from the patient following surgery for scoliosis and compared to three other archived samples to serve as a control. It was concluded that muscle was not affected by the CreaT mutation since no abnormalities were seen in creatine concentration, muscle histology, and electron transport chain activity. The authors suggested that there are different methods, as well as several factors, that allow creatine uptake in the muscle when compared to the brain. Creatine synthesis was also ruled out since synthesis has not been observed in skeletal muscle in humans nor in animal models. This 
research clarifies that muscle and brain respond differently to creatine transport.

Since meat is the primary dietary source for creatine, the examination of vegetarians may provide a unique aspect to creatine metabolism research. Watt et al. examined how five days of creatine supplementation affects vegetarians versus omnivores in regards to total creatine content and CreaT expression ${ }^{20}$. Results indicated that vegetarians had a lower initial total creatine concentration, and during supplementation, both groups significantly increased total creatine levels. However, vegetarians' total creatine content increased to a greater extent. Therefore, during a creatine loading protocol, vegetarians appear to possess a greater ability to take up creatine when compared to their omnivorous counterparts.

Previous research has concluded that there is no gender difference in the total creatine content in muscle, either before or after supplementation. Although this suggests that CreaT activity is unlikely to differ between genders, no research has directly investigated this. Murphy et al. chose to focus upon whether there is a difference between genders in CreaT mRNA and protein in healthy, young adults ${ }^{21}$. Two groups, separated by gender, had a muscle biopsy taken, which was then analyzed for CreaT mRNA, CreaT protein, and total creatine content. The results failed to show any differences between genders in neither total creatine content nor CreaT protein quantity, with CreaT protein expression greatest in type I fibers than in type II muscle fibers. Lastly, results concluded that there was an inverse relationship between total creatine content and CreaT protein content for females. It was noted that this relationship was also very close to being significant for males as well; after an outlier was removed, both genders proved statistically significant. Therefore, as total creatine content increases, the creatine transporter content decreases, and vice versa. This research exhibits the same indirect relationship that has been observed in previous animal models. The authors suggest that muscle fiber type needs to be taken into account for future research measuring CreaT expression, since type I fibers tend to have a greater abundance of CreaT protein.

Previous animal research has repeatedly shown a down regulation in CreaT expression following long-term creatine supplementation. It is argued that since the animal doses of creatine were much higher when equated to humans, downregulation of the CreaT may be very slight or nonexistent when applied to a typical moderate dosing regimen in human individuals. Tarnopolsky et al. chose to examine this particular issue in order to "determine whether a moderate-term (2 month) creatine monohydrate supplementation protocol would down-regulate the total amount of CreaT protein in young and elderly individuals participating in a resistance exercise training protocol ${ }^{22}$." Results concluded that intracellular creatine levels were significantly elevated among all groups. Additionally, no alteration in CreaT protein content was apparent with either creatine supplementation or exercise training. CreaT mRNA was not affected following acute creatine loading. In addition to Murphy's work, ${ }^{21}$ no difference between genders were observed in relation to CreaT protein or CreaT mRNA abundance. In conclusion, creatine supplementation with a simultaneous resistance training protocol effectively raised the intracellular creatine content and did not result in a decrease in CreaT protein or CreaT mRNA.

\section{RESPONDERS VS. NONRESPONDERS}

Most studies have reported an increase in intramuscular creatine levels with supplementation; however, variability does exist. This poses the possible scenario of "responders" versus "nonresponders" to creatine supplementation. It is hypothesized that much of this variability lies within the regulation and activity of the creatine transporter. Unfortunately, most of the limited creatine supplementation research conducted investigating the expression of CreaT has been through animal models, as previously noted.

Greenhaff et al observed that approximately 20$30 \%$ of participants following a creatine loading regime did not respond with an increase in intracellular creatine ${ }^{24}$. This was defined as 
resting total muscle creatine levels less than $10 \mathrm{mmol} / \mathrm{kg}$ following a 5 day creatine loading phase at $20 \mathrm{~g} / \mathrm{d}$. Responders were then classified as achieving a $20 \mathrm{mmol} / \mathrm{kg}$ increase following the loading phase. Recently, Syrotuik and Bell ${ }^{25}$ conducted a descriptive profile of individuals portraying Greenhaff's classification of "responder" and "non-responder" characteristics. Results of this study concluded that the responders generally: 1) possessed a lower initial quantity of intramuscular creatine and were able to absorb and take up a greater amount through supplementation; 2) had a greater percentage of type II fibers; 3) had a greater fiber cross sectional area; and 4) possessed more fat-free mass. This data suggests that an individual's biological profile may partially determine the efficacy of a creatine supplementation protocol.
A significant amount of literature has developed in regards to the most effective means to enhance creatine uptake. Adding creatine with a carbohydrate source has been observed to enhance uptake, primarily through the effect of an insulin response ${ }^{4,26}$. Additionally, some research in cell culture has indicated that combining creatine and sodium may additionally enhance creatine uptake via the manipulation of increasing the gradient in which the CreaT functions ${ }^{27}$. The current literature is very preliminary in relation to examining how creatine supplementation affects CreaT expression while concomitantly following a resistance training protocol. In conclusion, it is prudent that future research begin to examine CreaT expression due to creatine supplementation in humans in much the same way as in animal models.

\section{REFERENCES}

1. Mesa JLM, Ruiz JR, Gonzalez-Gross MM, et al. Oral creatine supplementation and skeletal muscle metabolism in physical exercise. Sports Med. 2002;32(14):903-944.

2. Chanutin A. The fate of creatine when administered to man. J Biol Chem. 1926;67:29-37.

3. Chanutin A. A study of the effect of creatine on growth and its distribution in the tissues of normal rats. J Biol Chem. 1927;75:547-557.

4. Harris RC, Soderlund K, Hultman E. Elevation of creatine in resting and exercised muscle of normal subjects by creatine supplementation. Clin Sci. 1992;83:367-374.

5. Persky AM, Brazeau GA, Hochhaus G. Pharmacokinetics of the dietary supplement creatine. Clin Pharmacokinet. 2003;42(6):557-574.

6. Snow RJ, Murphy RM. Creatine and the creatine transporter: A review. Mol Cell Biochem. 2001;224:169-181.

7. Speer O, Neukomm LJ, Murphy RM, et al. Creatine transporters: A reappraisal. Mol Cell Biochem. 2004;256/257:407-424.

8. Walzel B, Speer O, Zanolla E, et al. Novel mitochondrial creatine transport activity. Implications for intracellular creatine compartments and bioenergetics. J Biol Chem. 2002;277:37503-37511.

9. Guerrero-Ontiveros ML, Wallimann T. Creatine supplementation in health and disease. Effects of chronic creatine ingestion in vivo: Down-regulation of the expression of creatine transporter isoforms in skeletal muscle. Mol Cell Biochem. 1988; 184:427-437.

10. Fitch CD, Shields RP, Payne WF, et al. Creatine metabolism in skeletal muscle. Specificity of the creatine entry process. $J$ Biol Chem. 1968;243:2024-2027.

11. Murphy R, McConell G, Cameron-Smith D, et al. Creatine transporter protein content, localization, and gene expression in rat skeletal muscle. Am J Physiol Cell Physiol. 2001;280:C415-C422.

12. Wang W, Jobst MA, Bell B, et al. Cr supplementation decreases tyrosine phosphorylation of the CreaT in skeletal muscle during sepsis. Am J Physiol Endocrinol Metab. 2001;282:E1046-E1054.

13. Peral MJ, Garcia-Delgado M, Calonge ML, et al. Human, rat and chicken small intestinal $\mathrm{Na}^{+}-\mathrm{Cl}^{-}-$creatine transporter: Functional, molecular characterization and localization. Journal of Physiology. 2002;545(1):133-144.

14. Brault JJ, Terjung RL. Creatine uptake and creatine transporter expression among rat skeletal muscle fiber types. $A m J$ Physiol Cell Physiol. 2003;284:C1481-C1489.

15. Brault JJ, Abraham KA, Terjung RL. Muscle creatine uptake and creatine transporter expression in response to creatine supplementation and depletion. J Appl Physiol. 2003;94:2173-2180.

16. Pyne-Geithman GJ, DeGrauw TJ, Cecil KM, et al. Presence of normal creatine in the muscles of a patient with a mutation in the creatine transporter: A case study. Mol Cell Biochem. 2004;262:35-39.

17. DeGrauw TJ, Cecil KM, Byars AW, et al. The clinical syndrome of creatine transporter deficiency. Mol Cell Biochem. 2004;244:45-48.

18. Tarnopolsky MA, Parshad A, Walzel B. Creatine transporter and mitochondrial creatine kinase protein content in myopathies. Muscle and Nerve. 2000;24:682-688. 
19. Guerrero-Ontiveros ML, Wallimann T. Creatine supplementation in health and disease. Effects of chronic creatine ingestion in vivo: Down-regulation of the expression of creatine transporter isoforms in skeletal muscle. Mol Cell Biochem. 1998; 184:427-437.

20. Watt KKO, Garnham AP, Snow RJ. Skeletal muscle total creatine content and creatine transporter gene expression in vegetarians prior to and following creatine supplementation. International Journal of Sport Nutrition and Exercise Metabolism. 2004;14:517-531.

21. Murphy RM, Tunstall RJ, Mehan KA, et al. Human skeletal muscle creatine transporter mRNA and protein expression in healthy, young males and females. Mol Cell Biochem. 2003;244:151-157.

22. Tarnopolsky M, Parise G, Fu MH, et al. Acute and moderate-term creatine monohydrate supplementation does not affect creatine transporter mRNA or protein content in either young or elderly humans. Mol Cell Biochem. 2003;244:159-166.

23. Argov Z, Taivassalo T, De Stefano N, et al. Intracellular phosphates in inclusion body myositis $-\mathrm{A}^{31} \mathrm{P}$ magnetic resonance spectroscopy study. Muscle Nerve. 1998;21:1523-1525.

24. Greenhaff PL, Bodin K, Soderlund K, et al. Effect of oral creatine supplementation on skeletal muscle phosphocreatine resynthesis. Am J Physiol. 1994;266: E745-E730.

25. Syrotuik DG, Bell GJ. Acute creatine monohydrate supplementation: A descriptive physiological profile of responders vs. nonresponders. J Strength Cond Res. 2004;18(3):610-617.

26. Green AL, Hultman E, Macdonald IA, et al. Carbohydrate ingestion augments skeletal muscle creatine accumulation during creatine supplementation in humans. Am J Physiol. 1996;271:E821-826.

27. Odoom JE, Kemp GJ, and Radda GK. The regulation of total creatine content in a myoblast cell line. Mol Cell Biochem. 1996;158:179-188. 